\title{
“CHOKING UNDER PRESSURE” IN OLDER DRIVERS
}

\author{
Kuan-Hua Chen, Steven W. Anderson, Michelle L. Rusch, \\ Nazan S. Aksan, Jeffrey D. Dawson \& Matthew Rizzo \\ University of Iowa \\ Iowa City, Iowa, USA \\ Email: matthew-rizzo@uiowa.edu
}

\begin{abstract}
Summary: Aging can impair executive control and emotion regulation, affecting driver decision-making and behavior, especially under stress. We used an interactive driving simulator to investigate ability to make safe left-turns across oncoming traffic under pressure in 13 older (> 65 years old) and 16 middle-aged (35-56 years old) drivers. Drivers made left-turns at an uncontrolled intersection with moderately heavy oncoming traffic. Gaps between oncoming vehicles varied and increased gradually from $2 \mathrm{~s}$ to $10 \mathrm{~s}$. Drivers made two left-turns with a vehicle honking aggressively behind (pressure condition), and two left-turns without the honking vehicle (control condition). Results showed that middle-aged drivers made more cautious turning decisions under pressure (by waiting for larger and safer gaps, $p<.001$ ), but older drivers did not. Further, older driver turning paths deviated under pressure compared to the control condition $(p<.05)$, but the middle-aged group did not. Moreover, across all subjects, better executive function was significantly correlated with larger increases of accepted gap size from control to honking $(p<.01)$. The findings suggest that older drivers are more sensitive to traffic challenges from environmental pressure and that neural models of older driver performance and safety must factor in age-related changes in executive control and emotion processing.
\end{abstract}

\section{INTRODUCTION}

We investigated effects of aging on driver reaction to social pressure from a honking vehicle. Being honked at may cause annoyance, stress, anger or panic that interferes with safe decisionmaking and driving. To mitigate this threat the driver must minimize distracting negative emotions, focus on road ahead, appraise the situation and respond safely, e.g., determine that the honker is unreasonable and no correction is needed, or that the honker is dangerous and that it is prudent to yield the right of way. If regulatory processes fail, excess emotional arousal may lead a driver to make hasty and dangerous decisions (Janis \& Mann, 1977).

We hypothesized that increased age would be associated with poorer driving responses to pressure from a honking vehicle. Ability to stay safe under pressure can be influenced by many factors, including personality, fatigue, and age. Aging is associated with decline of neural systems for executive control and emotion regulation (Tucker et al., 2012; Mather, 2012; West, 1996). Underlying emotional regulation mechanisms in at risk older drivers can be safely tested in a driving simulator.

We designed a simulator drive that involved four left-turns across oncoming traffic. Gaps between successive oncoming vehicles gradually increased from 2 s to 10 s. The longer subjects 
waited, the larger (and safer) gaps they could choose from. Subjects made two left-turns with a vehicle honking behind (pressure condition) and two left-turns without honking (control condition). Skin conductance indexed driver emotion arousal. Size of gaps accepted for left-turns indexed effects of honking on driver risk-perception and decision-making. Maximum deviation from road midline (DFM) during turns indexed effects of honking on driver maneuvers. Neuropsychological tests including the Wisconsin Card Sorting Task (WCST) and Trail Making Test part B (TMT-B) indexed driver executive functions.

\section{METHODS}

\section{Subjects}

Thirteen older (age $M=77.62$, $S D=4.86$; 9 men and 4 women) and sixteen middle-aged (age $M$ $=46.13, S D=5.41 ; 8$ men and 8 women) active drivers from the Iowa City area participated. All had normal or corrected-to-normal vision and were free from psychiatric and neurological conditions. Table 1 shows that older and middle-aged drivers did not differ significantly in education or exposure to driving (days per week).

Table 1. Demographic background, neuropsychological test scores, and DULA dangerous driving index (four subscales)

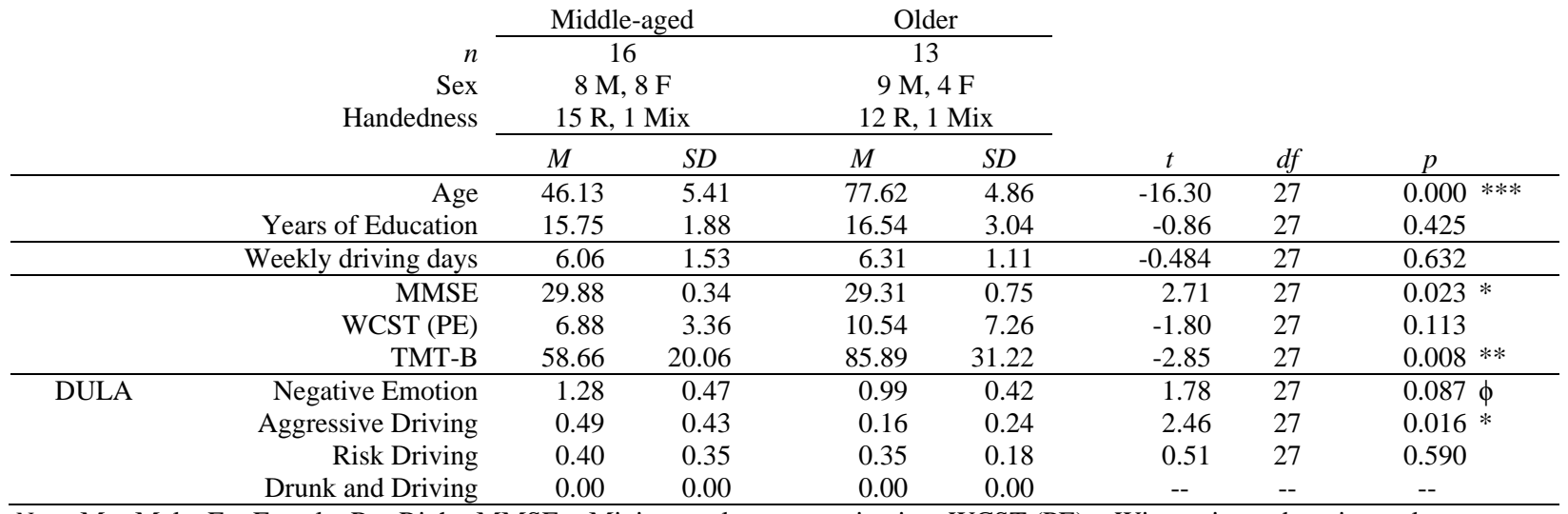

Note. $\mathrm{M}=$ Male; $\mathrm{F}$ = Female; $\mathrm{R}$ = Right; MMSE = Mini-mental state examination; WCST $(\mathrm{PE})=$ Wisconsin card sorting task (perseverative error); TMT-B = Trail making test part B (time to complete, in seconds); DULA = DULA dangerous driving index. $* * * p$ $<.001 ; * * p<.01 ;{ }^{*} p<.05 ;{ }^{\phi} p<.10$.

\section{Driving simulator}

We used the NADS MiniSim ${ }^{\mathrm{TM}}$ (version 1.8.3.3), a desktop-based driving simulator developed by the National Advanced Driving Simulator (NADS) and University of Iowa. The simulator has three 20 -inch display channels, providing a $110^{\circ}$ front-view (Figure 1) and a rear-view mirror.

\section{Left-turn Task}

The task comprised of four target and five non-target intersections. Subjects drove through the intersections sequentially during a non-stop primarily rural drive. In each target intersection, subjects had to make a left-turn across a stream of oncoming vehicles. Gaps between oncoming vehicles varied and gradually increased from 2 s to 10 s (e.g., 2, 2, 3, 2, 3, 4, 2, 4, 2, 2, 5). At two 
target intersections, a vehicle applied pressure by honking aggressively behind the drivers as they contemplated the left-turn. The other two target intersections had no honking vehicle behind the drivers (control condition). The order of target intersections was constant across all subjects (pressure, control, control, pressure). Five non-target intersections were embedded between target intersections, where subjects made a right-hand turn or a left-hand turn with no oncoming traffic or honking vehicle. Subjects were told that the goal of the task was simply to drive safely throughout the course.

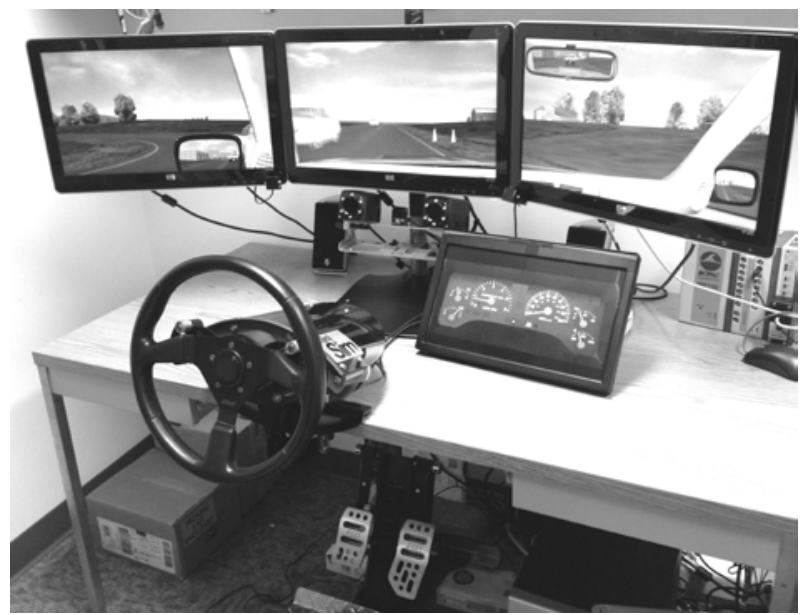

Figure 1. NADS MiniSim ${ }^{\mathrm{TM}}$ and a screenshot of the left-turn scenario

\section{Left-turn path analyses: Maximum deviation from midline (DFM)}

Figure 2 shows the geographic window for DFM analyses, which is from line B to line C. We calculated the longest distance from driver path to road midline (the dashed line) when drivers were between line B and C. Lane width was about 12 feet, and driving 12 feet or more off midline, put the driver on the shoulder or grass.

\section{Skin conductance recording and data processing}

For 26 subjects (10 older and 16 middle-aged), we measured skin conductance activity using a BIOPAC MP150 system (sampling rate $=1000 \mathrm{~Hz}$; data were offline reduced to $100 \mathrm{~Hz}$ for processing). Two electrodes were placed on the bottom of the left foot. Skin conductance artifacts were identified offline and were corrected using Ledalab software (Benedek \& Kaernbac, 2010). Ledalab also automatically identified significant skin conductance responses (SCRs; significant SCRs are the SCRs with trough-to-peak amplitudes higher than $0.01 \mathrm{uS}$ ) during each target intersection and calculated SCR magnitude (mean area under curve). Geographic window for SCR analyses was from line A to $5 \mathrm{~s}$ before subjects drove over line B (Figure 2). We normalized SCR data for each intersection by dividing each subject's SCR range over the whole task (Lykken, 1972).

\section{Neuropsychological tests and real-world dangerous driving index}

The Wisconsin Card Sorting Task (WCST; perseverative errors) and Trail Making Test part B (TMT-B; time to complete, in seconds) measured executive functions. Subjects self-reported the frequency of their dangerous driving behaviors on 28 question index covering four aspects of 
dangerous driving: Negative cognition and emotions, aggressive driving, risky driving, and drunk driving (DULA; Dula \& Ballard, 2003).

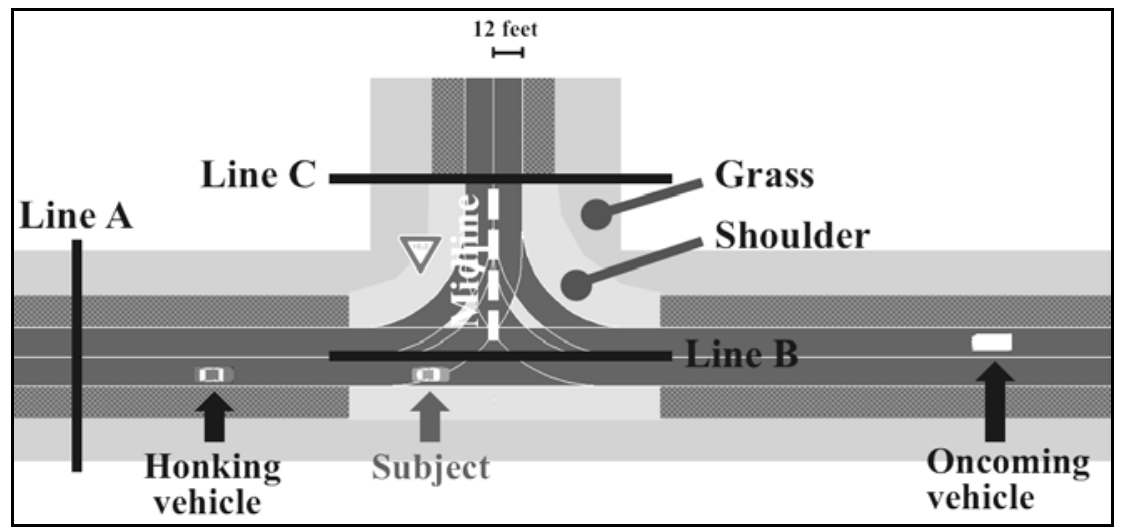

Figure 2. Analysis windows for maximum deviation from midline (DFM) and skin conductance response (SCR)

\section{Procedure}

All subjects provided informed consent for participation in compliance with the local Institutional Review Board. Most subjects completed their participation in two visits. During the first visit, subjects completed the Mini-Mental State Examination (MMSE), basic visual functions tests, and demographic and driving status questions. They also completed one to two practice drives in the simulator, depending on how well they adapted. The second visit started with another practice drive. SCR electrodes were then placed on the left foot. Instructions were explained verbally and also presented visually via PowerPoint presentation. Before the left-turn task, subjects completed an urban driving task that included multiple left- and right-turns without social pressure (data not reported here). The left-turn task started immediately after the urban section and lasted approximately 10 minutes. At the end of the drive, subjects completed questionnaires (e.g., DULA) and were debriefed on their study participation.

Neuropsychological data were collected for all middle-aged subjects and one older subject after completing driving tasks. The other 12 older subjects completed neuropsychological testing within eight months prior to this study. As all were healthy, stable, and neurologically normal, these data were considered representative of their cognitive status at the time of the driving testing.

\section{Statistical tests}

Major dependent variables included: SCR, size of accepted gaps, and DFM. To test within-group differences (pressure vs. control) on those variables, we calculated average responses within a person within a condition, and conducted paired $t$-tests separately for each driver group. To test between-group differences (of degree for change from control to pressure conditions), we calculated differences between pressure and control conditions (pressure - control) on the three major variables and independent-samples $t$ tests. For secondary dependent variables, including WCST, TMT-B, DULA subscales, and demographic variables, we conducted independentsamples $t$-tests to examine between-group differences. We also conducted Pearson correlations 
between neuropsychological tests and major dependent variables (on degree for change from control to pressure conditions).

\section{RESULTS}

\section{Skin conductance responses}

Figure 3 shows higher SCRs $(p<0.039)$ in the middle-aged drivers and a trend toward higher SCRs $(p<0.057)$ in older subjects in the honking compared to the control condition. The magnitude of SCR difference between the honking and control conditions did not differ significantly between the middle-aged and older driver groups $(p<0.762)$.

\section{Skin Conductance Response (SCR)}

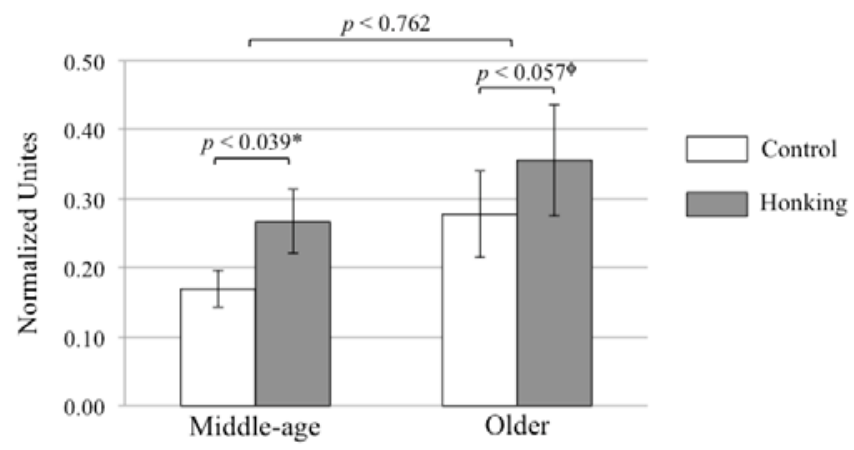

\section{Size of accepted gaps}

Figure 3. Skin conductance responses (SCRs; $\left.{ }^{*} p<.05 ;{ }^{\phi} p<.10\right)$

Figure 4A shows that gap size accepted by middle-aged subjects was larger under honking pressure than in the control condition $(p<0.007)$. This pattern was not observed in the older subjects $(p>0.157)$. No group difference in the extent of change (pressure - honking) was found $(p>0.40)$.

\section{Left-turn path - Maximum deviation from midline (DFM)}

Figure 4B shows that degree of deviation from road midline was unchanged across conditions in the middle-aged subjects ( $p>0.831$ ). In contrast, older subjects deviated significantly more under honking pressure compared to the control condition $(p<0.018)$. The differences in midline deviation between honking and control conditions were higher for older drivers compared to middle-aged drivers ( $p<0.015$; the interaction of group by control vs. pressure difference).

\section{Neuropsychological tests and DULA}

Older drivers had lower scores on the MMSE and TMT-B compared to middle-aged drivers ( $p$ s $<0.023$; Table 1). They also showed a trend toward worse performance in the WCST (higher $\mathrm{PE})(p<0.113)$. More intriguingly, across all drivers, WCST score (PE) correlated with changes in gap sizes accepted from control to honking conditions $(r=-0.473, p<.01)$, which means that better executive function tended to be correlated with larger increases of accepted gap size (from control to honking). This pattern was still significant after the control for age $(r=-.448, p<$ 
.025). No other significant correlation was found between neuropsychological tests and major dependent variables ( $p$ s > .354).

(A)

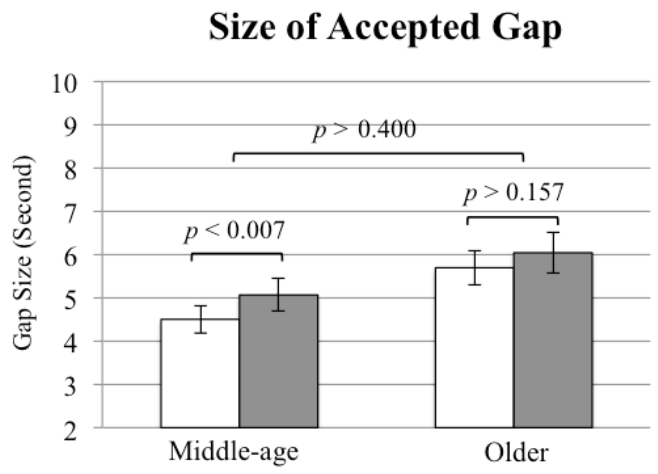

(B)

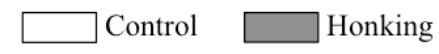

Max. Deviation from Road Midline

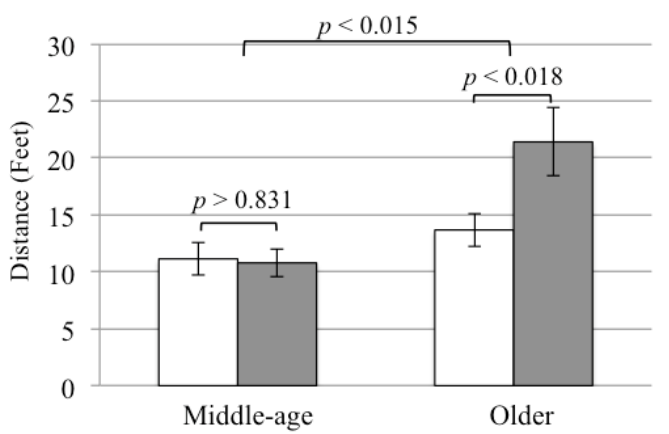

Figure 4. (A) Size of accepted gaps (B) Maximum deviation from road midline $\left({ }^{* *} p<.01 ;{ }^{*} p<.05\right)$

On the DULA, middle-aged subjects reported a higher frequency of aggressive driving ( $p<$ 0.016 ) and negative emotions ( $p<0.087$; marginally significant) in real-world driving compared to the older subjects.

\section{DISCUSSION}

In this study of older and middle-aged drivers, the middle-aged displayed more cautious decision-making under social pressure created by a honking driver, by waiting longer for larger and safer gaps when faced with making left-turns at uncontrolled intersections. One possibility for the increased caution under honking pressure was a metacognitive awareness by the drivers of the potential adverse influence of honking on driver decision-making. In this vein, some drivers reported that they felt stressed by honking and attempted to calm themselves to eliminate the effects of this stress on their driving. In addition to taking larger gaps, middle-aged drivers did not compromise vehicle control under pressure. Their turning trajectories in the honking condition were similar to those in the control condition. In contrast to middle-aged drivers, older drivers did not become more cautious by choosing to wait for longer and safer gaps. Moreover, their turning paths became worse as evidenced by greater deviation from the road midline under the honking pressure compared to the control condition, tantamount to "choking under pressure" (DeCaro et al., 2011).

These results fit with the findings of lower scores of older performers in executive functions conducted in the laboratory. In this study, we found that WCST score was associated with increased safety margins, choosing larger gaps in honking compared to non-honking condition. This finding suggests that resistance to honking pressure requires cognitive flexibility to switch attention from honking to the left-turn task. Decline in cognitive flexibility in older drivers (indexed by the WCST) might reduce their ability to redeploy attention from honking.

Results of the current experiment may also fit with findings of decreased emotional regulation ability in advancing age. Older individuals are reported to have a greater difficulty in cognitive reappraisal (Tucker et al., 2011). In this study, middle-aged drivers might re-appraise the left- 
turn situation in the context of the aggressive driver was unreasonable and dangerous. Therefore they showed more cautious gap acceptance and stable turning trajectories during honking. Under this same pressure however, the older drivers accepted potentially unsafe gaps and showed compromised turning paths.

We acknowledge the relatively small sample in this study. We note that "choking under pressure" may span a range of dysfunction, from upregulation to decrease in emotional reactivity. In one sense it may reflect an exaggerated reactivity (akin to increased startle) that may accompany neurodegeneration. Conversely it may reflect a general reduce of emotional sensation in older individuals (Mather, 2012).

In conclusion, the current evidence suggests that common driver-to-driver conflicts can disproportionally affect decision-making, performance and safety in drivers with age related cognitive decline. Factoring in age-related changes in executive control and emotional regulation should strengthen neural models of driving and interventions to improve older driver safety.

\section{ACKNOWLEDGEMENT}

This study was supported by Nissan Motor Company. We thank Andrew Veit, Chris Schwarz, David Heitbrink, Omar Ahmad, and Meiji Zhang in the National Advanced Driving Simulator for their support and advice on MiniSim ${ }^{\mathrm{TM}}$ hardware and software. We thank Katherine Read and Kelsey Thompson for collecting partial neuropsychological data, Samantha Edwards and Ruth Henson for assistance with human subjects issues, and Amy Johnson for pre-processing simulator data. We also thank all of our subjects for their participation.

\section{REFERENCES}

Benedek, M. \& Kaernbach, C. (2010). A continuous measure of phasic electrodermal activity. Journal of Neuroscience Methods, 190, 80-91.

DeCaro, M., Thomas, R., Albert, N., \& Beilock, S. (2011). Choking under pressure: Multiple routes to skill failure. Journal of Experimental Psychology: General, 140(3), 390-406.

Dula, C. S., \& Ballard, M. E. (2003). Development and evaluation of a measure of dangerous, aggressive, negative emotional, and risky driving1. Journal of Applied Social Psychology, 33(2), 263-282.

Janis, I. L., \& Mann, L. (1977). Decision making: A psychological analysis of conflict, choice, and commitment. New York: Free Press.

Lykken, D. T. (1972). Range correction applied to heart rate and to GSR data. Psychophysiology, 9(3), 373-379.

Mather, M. (2012). The emotion paradox in the aging brain. Annals of the New York Academy of Sciences, 1251(1), 33-49.

Tucker, A. M., Feuerstein, R., Mende-Siedlecki, P., Ochsner, K. N., \& Stern, Y. (2012). Double dissociation: Circadian off-peak times increase emotional reactivity; aging impairs emotion regulation via reappraisal. Emotion, 12(5), 869-874.

West, R. L. (1996). An application of prefrontal cortex function theory to cognitive aging. Psychological Bulletin, 120(2), 272-292. 